\title{
Nekrasov-Shatashvili limit of the 5D superconformal index
}

\author{
Constantinos Papageorgakis, ${ }^{1, *}$ Alessandro Pini, ${ }^{2, \dagger}$ and Diego Rodríguez-Gómez ${ }^{2, \hbar}$ \\ ${ }^{1}$ CRST and School of Physics and Astronomy Queen Mary University of London, \\ London E1 4NS, United Kingdom \\ ${ }^{2}$ Department of Physics, Universidad de Oviedo Avda. Calvo Sotelo 18, 33007 Oviedo, Spain
}

(Received 6 June 2016; published 8 August 2016)

\begin{abstract}
We consider the Nekrasov-Shatashvili limit of the five-dimensional (5D) superconformal index and propose a novel prescription for selecting the finite contributions. Applying the latter to various examples of U(1) theories, we find that the 5D Nekrasov-Shatashvili index can be reproduced using recent techniques of Córdova and Shao, who related the 4D Schur index to the Bogomol'nyi-Prasad-Sommerfield (BPS) spectrum of the theory on the Coulomb branch. In this picture, the 5D instanton solitons are interpreted as additional flavor nodes in an associated 5D BPS quiver.
\end{abstract}

DOI: $10.1103 /$ PhysRevD.94.045007

\section{INTRODUCTION AND SUMMARY}

The superconformal index has proven to be an important tool in the study of superconformal field theories (SCFTs) in diverse dimensions [1,2]. In some cases, interesting limits of the index have been devised, which isolate contributions from particular subsets of operators and provide information about its different phases; see e.g Ref. [3]. Limits of the index also help in identifying algebraic structures hidden within special subsectors of the theory, a fact which has been put to remarkable effect in four and six dimensions [4].

In a closely related direction, recent work [5] established a connection between the so-called Schur limit of the fourdimensional (4D) index on the one hand ${ }^{1}$ and a certain algebraic quantity associated with the BPS spectrum of particles on the Coulomb branch on the other-the trace of the Kontsevich-Soibelman (KS) operator-for a convincing number of $4 \mathrm{D} \mathcal{N}=2 \mathrm{SCFTs}^{2}$; see also Ref. [8] for generalizations. In this fashion, one demonstrates that, for specific BPS subsectors, the operator spectrum of an SCFT is directly related to the particle spectrum of the same theory in a phase where the conformal symmetry has been broken.

In this paper, we would like to import some of these results to five-dimensional SCFTs [9-12]. Our first objective will be to define a limit of the five-dimensional (5D) superconformal index by turning off one of the two $\Omega$-deformation parameters ${ }^{3}$; this is the limit first considered by Nekrasov and Shatashvili (NS) in a four-dimensional

\footnotetext{
*c.papageorgakis@qmul.ac.uk

†pinialessandro@uniovi.es

\#d.rodriguez.gomez@uniovi.es

${ }^{1}$ Recent exact results on the 4D Schur index include Ref. [6].

${ }^{2}$ For an alternative calculation of the Schur index for ArgyresDouglas theories, see Ref. [7].

${ }^{3}$ Since the precise operator spectrum of the interacting 5D UV theories is unknown, one usually works with the realization of the index as a supersymmetric partition function on $S^{4} \times S^{1}$ with twisted boundary conditions for the various fields.
}

context [13]. Its naive implementation leads to a singular index, which calls for a prescription on how to extract the finite parts. This problem can in principle be addressed in a way similar to the original NS limit of [13]. However, the direct 5D extension of that recipe leads to a function of which the fugacity expansion does not necessarily involve integer coefficients. In turn, we propose a different 5D regularization which results in a fugacity expansion with integer coefficients for arbitrary gauge groups. In the Abelian case, our regularization clearly isolates contributions from states localized on a four-dimensional subspace of the Euclideanized spacetime. Moreover, it reproduces, at least for the perturbative sector, the large-orbifold limit of the gauge theory index of Ref. [14]. The latter effectively reduces the space down to a 4D geometry —of the form $M_{3} \times S^{1}$ where the contributions of vector and hypermultiplets become identical to the 4D Schur index and may hint toward an interesting connection with Ref. [4]. Although our limit does not lead to a counting of states preserving a larger fraction of supersymmetry, it does lead to a factorization of the index into a "holomorphic" and "antiholomorphic" part for general 5D SCFTs. This factorization is reminiscent of the work of Iqbal and Vafa [15], where it also appeared as the starting point for connecting the 5D BPSparticle degeneracy ${ }^{5}$ to the index, using the topological string.

With this last point in mind, our second objective will be to relate the NS limit of the 5D index to the work of Ref. [5]. For a number of Abelian examples, we will show that the NS index can be reproduced by the trace of the KS operator for a " $5 \mathrm{D}$ BPS quiver." This quiver can be constructed straightforwardly by assigning a node for each "partonic BPS state.", This involves a node corresponding to the instanton-soliton parton of the 5D theory, as well as a

\footnotetext{
${ }^{4}$ Interesting limits of the 4D index with additional superymmetry were originally considered in Ref. [3].

${ }^{5}$ Note that in $5 \mathrm{D}$ there also exist BPS strings.
} 
node for each of the possible $N_{f}$ hypermultiplets of the theory. The construction and study of the 5D BPS quiver for non-Abelian theories, and their possible connection to the NS index, is a question that we will leave open for future investigation. However, our Abelian results can already be thought of as a check of the proposal of Ref. [15], for a particular subsector of five-dimensional theories.

The rest of this article is organized as follows. In Sec. II, we will present the details of the NS limit for the 5D index, after briefly reviewing some background material necessary for our discussion. Then, in Sec. III, we will introduce the algebraic tools of Ref. [5] and use them to recover our index for $\mathrm{U}(1)$ theories with different matter content and values of the Chern-Simons coefficient. We will also discuss some directions for generalizing these results to non-Abelian gauge groups.

\section{NEKRASOV-SHATASHVILI LIMIT OF THE 5D INDEX}

\section{A. Generalities}

The superconformal index in five dimensions was first defined in Ref. [2] and computed using supersymmetric localization [16] for a variety of $\mathcal{N}=1$ theories in Ref. [17]. Recall that, using a Verma module construction, one can obtain all irreducible representations of the 5D superconformal algebra (SCA) $F(4)$ from irreducible representations of the maximal compact subalgebra $\mathfrak{g} \mathfrak{o}(2)_{E} \oplus \mathfrak{g} \mathfrak{o}(5) \oplus \mathfrak{g} \mathfrak{t}(2)_{R}$. The latter are labelled by strings of quantum numbers denoting the highest weight state $\left\{\epsilon_{0}, R, h_{1}, h_{2}\right\}$, where $h_{1}, h_{2}$ are the Cartan generators of $\mathfrak{g} \mathfrak{o}(5),{ }^{7}$ while $\epsilon_{0}$ is the scaling dimension measured by the charge under $\mathfrak{s} \mathfrak{o}(2)_{E}$. Finally, the $\mathfrak{s} \mathfrak{t}(2)_{R}$ Cartan generator is denoted by $R^{8}$

In the radial quantization of the theory, where $S=Q^{\dagger}$, and for a particular choice of supercharge, ${ }^{9}$ one can define

$$
\delta:=\{Q, S\}=\epsilon_{0}-h_{1}-h_{2}-3 R,
$$

which is a positive-definite quantity. The index is a partition function counting operators transforming in irreducible representations of the subalgebra of the SCA that (anti) commute with the above $Q, S$ (these are $\frac{1}{8}$-BPS) and hence also $\delta$-or equivalently, Irreducible representations of the commutant of $(Q, S, \delta)$ of the 5D SCA. It is straightforward to see that $h_{1}+R$ and $h_{2}+R$ commute with the above

\footnotetext{
${ }^{6}$ By this, we mean states with the lowest possible charges, i.e. ones that cannot be written as bound states of any other states.

${ }^{7}$ These are related to the $\Omega$-deformation parameters $\epsilon_{1}, \epsilon_{2}$ in a simple way.

${ }^{8}$ As is common in the literature, we will use the same symbols for the Cartan generators and the corresponding charges, depending on the context.

${ }^{9}$ We follow the conventions and choices of Ref. [17].
}

choice of $\delta$, and as a result the most general, or "refined," index with respect to the supercharge $Q$ is given by $[2,17,18]$

$$
I=\operatorname{Tr}_{\mathcal{H}_{\delta=0}}(-1)^{F} p^{h_{1}+R} q^{h_{2}+R} \prod_{a} w_{a}^{\mathfrak{Q}_{a}} \mathbf{q}^{k} .
$$

Here, the trace is taken over the Hilbert space of $\delta=0$ operators, $F=2 h_{1}$ is the fermion number operator, $p$ and $q$ are fugacities keeping track of the elements of the commutant, and the $w_{a}$ are additional fugacities for commuting charges $\mathfrak{Q}_{a}$, corresponding to possible global/gauge symmetries. One such commuting charge corresponds to a topological U(1) symmetry which is always present in the examples we are interested in: 5D gauge theories possess a conserved current, $* J=\frac{1}{8 \pi^{2}} \operatorname{tr}(F \wedge F)$, and their spectrum contains instanton solitons, charged under the associated symmetry. This global symmetry plays an important role in five dimensions, where SCFTs with very interesting properties exist [9]; in many cases, it can combine with and enhance other symmetries (flavor, Lorentz); see e.g. Refs. $[17,19,20]$. Indeed, one can also include a fugacity $\mathfrak{q}$ in the index (2.2), which keeps track of the instanton charge $k$, where $|\mathfrak{q}|=1$.

Via the state-operator map, the 5D index can alternatively be evaluated by a Euclidean path integral on $S^{4} \times S^{1}$ with twisted boundary conditions for the various fields according to their charges $[2,17,21]$. The index then counts $\frac{1}{8}$-BPS states for the theory on the sphere. This functional integral can be evaluated in the IR theory ${ }^{10}$ using localization [17], and the answer reduces to a gauge-group integral over the product of perturbative and nonperturbative contributions, schematically

$$
I=\int[\mathrm{d} U] Z_{\text {pert }}^{S^{4}} Z_{\text {nonpert }},
$$

with $[\mathrm{d} U]$ the unit-normalized Haar measure. The nonperturbative factor can be written as

$$
Z_{\text {nonpert }}=\left|Z_{\mathrm{Nek}}\right|^{2} \text {, }
$$

where $Z_{\mathrm{Nek}}$ is the Nekrasov instanton partition function [24]. The perturbative contribution is a modular quantity built out of the weak-coupling multiplets. The vector-multiplet and hypermultiplet contributions are given by

$$
I_{V, H}=\operatorname{PE}\left[f_{V, H}\right],
$$

where PE refers to the plethystic exponential. The so-called single-letter indices appearing above in turn read ${ }^{11}$

\footnotetext{
${ }^{10}$ For a generic SCFT on $\mathbb{R} \times S^{4}$, it is possible to turn on supersymmetrically a position-dependent Yang-Mills coupling, interpolating between the SCFT and the IR gauge theory $[22,23]$.

${ }^{11}$ For definiteness, we will assume that the hypermultiplet is in the fundamental of the gauge group.
} 


$$
\begin{aligned}
& f_{V}=-\frac{p+q}{(1-p)(1-q)} \chi_{\mathrm{Adj}}, \\
& f_{H}=\frac{\sqrt{p q}}{(1-p)(1-q)}\left(\chi_{\square}+\chi_{\bar{\square}}\right),
\end{aligned}
$$

with $\chi_{\mathcal{R}}$ denoting the character of a given representation $\mathcal{R}$.

\section{B. NS index}

Having set the stage, we would like to investigate whether there exist limits of the index (2.2) which only receive contributions from certain sectors of the theory, as e.g. is the case in 4D [3]. Note that, as opposed to other dimensions, the 5D index only depends on two fugacities. Moreover, these correspond to Cartans of SU(2) symmetries, a fact which underlies the $(p, q) \leftrightarrow\left(q^{-1}, p^{-1}\right)$ and $(p, q) \leftrightarrow(q, p)$ invariance of the index; cf. Eq. (2.6). Thus, it is hard to imagine nontrivial regular limits as in Ref. [3]. Yet, this does not exclude interesting singular limits. In particular, following [13], we will focus on the NS limit of the index. Generically, the NS limit involves sending one of the two $\Omega$-deformation parameters to zero, $\epsilon_{1} \rightarrow 0$ while keeping the other one, $\epsilon_{2}$, fixed. These parameters are chemical potentials for rotations in two real planes, $\mathrm{SO}(2)_{\epsilon_{1}} \times \mathrm{SO}(2)_{\epsilon_{2}} \subset \mathrm{SO}(5)$, and related to our choice of fugacities through $p=e^{-\epsilon_{1}}$ and $q=e^{-\epsilon_{2}}$. Hence, one can naively implement the NS limit directly at the level of the index, by considering

$$
p \rightarrow 1 \text { and } q \rightarrow \text { fixed. }
$$

Although this definition is natural, it leads to divergences as can be immediately seen by applying it to the perturbative contributions (2.6). We therefore need to put forward a modified definition for taking the NS limit of the 5D index, which leads to finite contributions.

Toward that end, we follow Ref. [18] and rewrite the index of the full theory on $S^{4} \times S^{1}$ in terms of two "hemisphere indices" on $D^{4} \times S^{1}$ with Dirichlet boundary conditions, where $D^{4} \subset S^{4}$ is half the sphere. The hemisphere index is in turn defined by

$$
I I=Z_{\mathrm{pert}}^{D^{4}} Z_{\mathrm{Nek}}
$$

For the example of a single vector multiplet and a hypermultiplet in the fundamental representation, the perturbative piece reads

$Z_{\mathrm{pert}}^{D^{4}}=\mathrm{PE}\left[-\frac{p+q}{(1-p)(1-q)} \chi_{\mathrm{Adj}}+\frac{\sqrt{p q}}{(1-p)(1-q)} \chi_{\square}\right]$,

where the gauge symmetry of the full index on $S^{4} \times S^{1}$ is to be understood as a global boundary symmetry.
The full index is then computed by combining two such contributions and gauging the appropriate diagonal subgroup of said global symmetries to obtain

$$
I=\left(I_{V}^{4 D}\right)^{r} \int[\mathrm{d} U] I I \bar{I} I,
$$

where the overline implies that one inverts all gauge/flavour fugacities. The term

$$
I_{V}^{4 \mathrm{D}}=\mathrm{PE}\left[-\frac{p}{1-p}-\frac{q}{1-q}\right]
$$

is a purely four-dimensional $\mathcal{N}=1$ vector-multiplet contribution coming from the boundary, and $r=\operatorname{rank}(G)$ is the gauge group rank.

We are now in the position to define the NS index as follows,

$$
\begin{aligned}
& \text { NS index: } I I^{\mathrm{NS}}\left(z_{i}, \mathfrak{q} ; q\right) \\
& \quad:=\mathrm{PE}\left[\lim _{p \rightarrow 1}(1-p) \mathrm{PE}^{-1}\left[I I\left(z_{i}, \mathfrak{q} ; p, q\right)\right],\right.
\end{aligned}
$$

such that

$$
I^{\mathrm{NS}}(\mathfrak{q} ; q):=\int[\mathrm{d} U] I I^{\mathrm{NS}}\left(z_{i}, \mathfrak{q} ; q\right) \overline{I I^{\mathrm{NS}}\left(z_{i}, \mathfrak{q} ; q\right)}
$$

Note that we have stripped off the (divergent in this limit) factors of $I_{V}^{4 \mathrm{D}}$. We will come back to this below.

We stress that this definition of the NS limit is different from other versions where the PE in (2.12) is traded for a standard exponential and results in a function of which the fugacity expansion does not necessarily involve integer coefficients; see Refs. [13,25]. On the other hand, Eq. (2.13) does admit an expansion with integer coefficients, due to the use of the PE.

In the above, the $z_{i}, i=1, \ldots, r$, are gauge/global symmetry fugacities, and the plethystic logarithm, $\mathrm{PE}^{-1}$, is the inverse of the plethystic exponential, defined as

$$
\mathrm{PE}^{-1}[g(t)]:=\sum_{n=1}^{\infty} \frac{\mu(n)}{n} \log \left[g\left(t^{n}\right)\right],
$$

with $\mu(n)$ the Möbius function. This factorization of the superconformal index in the NS limit is reminiscent of the discussion in Ref. [15], where the full index was calculated using the refined topological vertex formalism and related to the counting of BPS states on the Coulomb branch of the theory. We will see in the next section that the relationship to $5 \mathrm{D}$ BPS quivers can be quantified for $G=\mathrm{U}(1)$ through the formalism of Ref. [5]. 


\section{Perturbative NS limit}

Since our prescription for the NS limit (2.12) factorizes over the perturbative and nonperturbative contributions, let us first look at the former. From (2.9), it is straightforward to deduce that

$$
Z_{\mathrm{pert}}^{D^{4} \mathrm{NS}}=\mathrm{PE}\left[-\frac{q}{(1-q)} \chi_{\mathrm{Adj}}+\frac{\sqrt{q}}{(1-q)} \chi_{\square}\right],
$$

and consequently if we only focus on the perturbative sector,

$$
\begin{aligned}
\int & {[\mathrm{d} U] Z_{\mathrm{pert}}^{D^{4}, \mathrm{NS}} \overline{Z_{\mathrm{pert}}^{D^{4}, \mathrm{NS}}} } \\
& =\int[\mathrm{d} U] \mathrm{PE}\left[-\frac{2 q}{(1-q)} \chi_{\mathrm{Adj}}+\frac{\sqrt{q}}{(1-q)}\left(\chi_{\square}+\chi_{\square}\right)\right] .
\end{aligned}
$$

This is tantamount to projecting out states with a nontrivial $x_{++}$dependence, as can be seen by taking the NS limit directly on the full 5D single-letter indices.

This requires an equivalent prescription for which it is convenient to introduce fugacities $x=\sqrt{p q}, y=\sqrt{q / p}$. Note that, after performing this substitution in Eq. (2.2), the exponents of the $x$ and $y$ fugacities are given respectively by $h_{1}+h_{2}+2 R=2 j_{1}+2 R$ and $-h_{1}+h_{2}=-2 j_{2}$. In terms of these, the NS index for the hypermultiplet can be implemented by taking $y \rightarrow x$. More precisely,

$$
f_{H}^{\mathrm{NS}}=\lim _{\epsilon_{1} \rightarrow 0} \epsilon_{1} f_{H}\left(x, x\left(1+\epsilon_{1}\right)\right) .
$$

In this fashion, the NS index picks out the coefficient of the $\frac{1}{\epsilon_{1}}$ pole in the naive $\epsilon_{1} \rightarrow 0$ limit of $f_{H}$. Recall that for the free hypermultiplet the single particle index $f_{H}$ can be understood in terms of letter counting using the state-operator map [17]. Using Table I, one immediately sees that $f_{H}$ contains operators made out of letters of the form $\partial_{+ \pm}^{m} \mathcal{O}$; here, $\mathcal{O}$ is a scalar or fermionic component of the hypermultiplet, and the derivatives are responsible for the factor $(1-p)(1-q)=$ $(1-x y)\left(1-\frac{x}{y}\right)$ appearing in the denominator of (2.6). In the limit $\epsilon_{1} \rightarrow 0$, one such derivative becomes of zero weight. This results in a divergence in the limit $y \rightarrow x$, originating from an unrefinement in the index which now counts letters containing arbitrary powers of $\partial_{++}$with the same weight

TABLE I. The letters in the hypermultiplet and their respective charges.

\begin{tabular}{lccc}
\hline \hline & $\epsilon_{0}$ & $\left(j_{1}, j_{2}\right)$ & $R$ \\
\hline$q$ & $\frac{3}{2}$ & $(0,0)$ & $\pm \frac{1}{2}$ \\
$\psi$ & 2 & $\left( \pm \frac{1}{2}, 0\right) \oplus\left(0, \pm \frac{1}{2}\right)$ & 0 \\
$\partial$ & 1 & $\left( \pm \frac{1}{2}, \pm \frac{1}{2}\right) \oplus(0,0)$ & 0 \\
\hline \hline
\end{tabular}

(zero). Defining the NS index through selecting the pole in (2.17) is tantamount to only accounting for the contribution with no derivatives.

Somewhat surprisingly, the vector-multiplet piece can also be given an IR-operator interpretation. In such a scenario, one can understand the single-letter vector-multiplet contribution as arising from components of the gaugino plus a tower of infinitely many derivatives. In the limit $\epsilon_{1} \rightarrow 1$, not only the weight of a derivative but also one of the components of the gaugino become zero. These translate into singularities of the index, and our prescription amounts to regularizing them by discarding zero-weight letters.

Hence, at the level of implementation, the following single-letter functions can be used for the perturbative contributions in the NS limit:

$f_{V}^{\mathrm{NS}}=-\frac{2 q}{(1-q)} \chi_{\mathrm{Adj}}, \quad f_{H}^{\mathrm{NS}}=\frac{\sqrt{q}}{(1-q)}\left(\chi_{\square}+\chi_{\bar{\square}}\right)$.

We highlight that these single-letter terms are precisely the vector and hypermultiplet single-letter index contributions for the perturbative sector of $\mathcal{N}=2$ four-dimensional theories in the Schur limit $[3,6]$, which may hint at a connection with the results of Ref. [4]. It is also interesting to observe that the large-orbifold limit of Ref. [14] also led to perturbative contributions identical to those of the $4 \mathrm{D}$ Schur index. ${ }^{12}$

All in all, in the perturbative sector our NS limit discards states with a dependence on the $x_{++}$direction on $D^{4}$, along with the boundary $\mathcal{N}=1$ vector-multiplet contributions $I_{V}^{4 D}$. This is equivalent to using the single-letter expressions (2.18) directly in (2.5). We will next see that this interpretation extends to the nonperturbative sector for Abelian theories.

\section{Nonperturbative NS limit}

The result of the prescription (2.12) on the nonperturbative piece is somewhat more involved. This is due to the fact that, with the exception of the Abelian case, the Nekrasov partition function cannot be written as a PE of single-letter contributions but is evaluated as an expansion in powers of the instanton fugacity $\mathfrak{q}$,

$$
Z_{\mathrm{Nek}}=\sum_{k=0}^{\infty} \mathfrak{q}^{k} Z_{\mathrm{Nek}}^{(k)} \quad \text { with } \quad Z_{\mathrm{Nek}}^{(0)}=1 .
$$

We will henceforth assume that the NS limit commutes with the instanton expansion and then use this along with (2.12) to get

\footnotetext{
${ }^{12}$ Recall that Ref. [14] considered the 5D theory on $S^{4} / \mathbb{Z}_{n} \times$ $S^{1}$ in the large- $n$ limit. This effectively dimensionally reduced the space down to a (singular) 4D geometry.
} 


$$
\begin{aligned}
Z_{\mathrm{Nek}}^{\mathrm{NS}}\left(z_{i}, \mathfrak{q} ; q\right)= & \operatorname{PE}\left[\lim _{p \rightarrow 1}(1-p) \mathrm{PE}^{-1}\left[\sum_{k=0}^{\infty} \mathfrak{q}^{k} Z_{\mathrm{Nek}}^{(k)}\left(z_{i} ; p, q\right)\right]\right] \\
= & \operatorname{PE}\left[\lim _{p \rightarrow 1}(1-p) \mathrm{PE}^{-1}\left[1+\mathfrak{q} Z_{\mathrm{Nek}}^{(1)}\left(z_{i} ; p, q\right)+\mathfrak{q}^{2} Z_{\mathrm{Nek}}^{(2)}\left(z_{i} ; p, q\right)+O\left(\mathfrak{q}^{3}\right)\right]\right] \\
= & \operatorname{PE}\left[\operatorname { l i m } _ { p \rightarrow 1 } ( 1 - p ) \left(\mathfrak{q} Z_{\mathrm{Nek}}^{(1)}\left(z_{i} ; p, q\right)\right.\right. \\
& \left.+\mathfrak{q}^{2}\left(Z_{\mathrm{Nek}}^{(2)}\left(z_{i} ; p, q\right)-\frac{1}{2} Z_{\mathrm{Nek}}^{(1)}\left(z_{i} ; p, q\right)^{2}-\frac{1}{2} Z_{\mathrm{Nek}}^{(1)}\left(z_{i}^{2} ; p^{2}, q^{2}\right)+O\left(\mathfrak{q}^{3}\right)\right)\right] \\
= & 1+\mathfrak{q}_{p \rightarrow 1}(1-p) Z_{\mathrm{Nek}}^{(1)}\left(z_{i} ; p, q\right) \\
& +\mathfrak{q}^{2} \lim _{p \rightarrow 1}\left((1-p)\left(Z_{\mathrm{Nek}}^{(2)}\left(z_{i} ; p, q\right)-\frac{1}{2} Z_{\mathrm{Nek}}^{(1)}\left(z_{i} ; p, q\right)^{2}-\frac{1}{2} Z_{\mathrm{Nek}}^{(1)}\left(z_{i}^{2} ; p^{2}, q^{2}\right)\right)\right. \\
& \left.+(1-p)^{2} \frac{Z_{\mathrm{Nek}}^{(1)}\left(z_{i} ; p, q\right)^{2}}{2}+\left(1-p^{2}\right) \frac{Z_{\mathrm{Nek}}^{(1)}\left(z_{i}^{2} ; p^{2}, q^{2}\right)}{2}\right)+O\left(\mathfrak{q}^{3}\right) \\
= & \sum_{k=0}^{\infty} \mathfrak{q}^{k} Z_{\mathrm{Nek}}^{\mathrm{NS},(k)}\left(z_{i} ; q\right) .
\end{aligned}
$$

This proposal is obviously applicable to the case of $G=\mathrm{U}(1)$, where, as we will see shortly, the instanton expansion can be explicitly resummed into a PE. For example, for a pure U(1) theory, one has

$$
Z_{\text {nonpert }}=\operatorname{PE}\left[\frac{\sqrt{p q}}{(1-p)(1-q)}\left(\mathfrak{q}+\mathfrak{q}^{-1}\right)\right]
$$

In that context, the NS limit once again explicitly counts states which do not have any dependence on the $x_{++}$ direction. ${ }^{13}$ However, the definition (2.20) also makes sense for the case of non-Abelian gauge groups, where $Z_{\mathrm{Nek}}^{(k)}$ can be expanded in $q$ to yield terms with integer coefficients, as expected for an index. We have explicitly checked this to sufficiently high order for $G=\mathrm{SU}(2)$.

As raised above, we should emphasize that a version of the NS limit for the Nekrasov partition function has already been considered in Ref. [25], along the lines of Ref. [13]. This is a different limit from the one discussed here, insofar as it involves replacing plethystic exponentials with exponentials and plethystic logarithms with logarithms. Our motivation for (2.12) stems from requiring finite coefficients in the fugacity expansion and mirroring the definition of the 4D limits of Ref. [3], which act directly on the single-letter indices.

\footnotetext{
${ }^{13}$ One can also ascribe an IR-operator interpretation to the Abelian instanton partition function, as the PE of single-letter contributions from instanton operators $[19,23]$.
}

\section{KONTSEVICH-SOIBELMAN OPERATORS AND BPS QUIVERS}

Having provided our definition for the NS index, one can establish a connection with Ref. [5]. In that reference-see also Ref. [8] —it was conjectured that the 4D Schur index of a rank- $r$ theory can be recovered in terms of quantities associated with the BPS quiver of the theory [26] through

$$
I_{\mathrm{KS}}=(q)_{\infty}^{2 r} \operatorname{Tr}[\mathcal{O}],
$$

where the Pochhammer symbol is defined as

$$
(q)_{0}=1, \quad(q)_{n}=\prod_{k=1}^{n}\left(1-q^{k}\right) .
$$

Here, the quantity $\mathcal{O}$ is the KS operator associated with the BPS quiver of the four-dimensional gauge theory. Such a theory contains a set of BPS particles on the Coulomb branch labelled by a vector $\gamma$ in the charge lattice $\Gamma$. Then, for each $\gamma$, one introduces a formal variable $X_{\gamma}$ obeying a quantum torus algebra,

$$
X_{\gamma} X_{\gamma^{\prime}}=q^{\frac{\left\langle\gamma \gamma^{\prime}\right\rangle}{2}} X_{\gamma+\gamma^{\prime}}=q^{\left\langle\gamma, \gamma^{\prime}\right\rangle} X_{\gamma^{\prime}} X_{\gamma},
$$

where $\langle\cdot, \cdot\rangle$ is the (integer) Dirac pairing of charges in the lattice $\Gamma$, which can be read off from the BPS quiver. In terms of these $X_{\gamma}$, the KS operator can be explicitly written as

$$
\mathcal{O}=\prod_{\gamma} E_{q}\left(X_{\gamma}\right)
$$


where $E_{q}$ is the $q$-exponential function

$$
E_{q}(z)=\prod_{i=0}^{\infty}\left(1+q^{i+\frac{1}{2}} z\right)^{-1}=\sum_{n=0}^{\infty} \frac{\left(-q^{\frac{1}{2}} z\right)^{n}}{(q)_{n}} .
$$

For a theory without flavor, the trace of the quantum torus algebra is defined by its action on the formal variables $X_{\gamma}$,

$$
\operatorname{Tr}\left[X_{\gamma}\right]= \begin{cases}1 & \gamma=0 \\ 0 & \text { otherwise }\end{cases}
$$

and extending linearly. For theories with flavor, there exist flavor charge vectors $\gamma_{f}$, which have zero Dirac pairing with all other $\gamma^{\prime} \in \Gamma,\left\langle\gamma_{f}, \gamma^{\prime}\right\rangle=0$. Moreover, the definition of the trace needs to be modified to

$\operatorname{Tr}\left[X_{\gamma}\right]=\left\{\begin{array}{lll}\prod_{i} \operatorname{Tr}\left[X_{\gamma_{f_{i}}}\right]_{i}^{f_{i}(\gamma)} & \left\langle\gamma, \gamma^{\prime}\right\rangle=0 \quad \forall \gamma^{\prime} \in \Gamma \\ 0 & \text { otherwise }\end{array}\right.$

where $\gamma_{f_{i}}$ is an integral basis of flavor charges and $f_{i}(\gamma)$ is the flavor charges of $\gamma$. The $\operatorname{Tr}\left[X_{\gamma_{f_{i}}}\right]$ are free quantities that are to be identified with the flavor fugacities appearing in the index. Using the above machinery, the 4D Schur index can be read off from the BPS quiver [5].

In view of the similarities between the NS limit of the 5D index discussed above and the Schur index for an $\mathcal{N}=24$ D theory with the same number of vector and hypermultiplets, it is natural to wonder whether a decomposition in terms of 5D BPS quiver data also exists. In fact, Iqbal and Vafa have used the topological string [15] to argue that the 5D BPS-particle spectrum reproduces the superconformal index.

We will next provide a simple but concrete realization of this idea, relating the NS index to the trace of the KS operator for a number of Abelian examples. At this point, we should make it clear that there exist no nontrivial Abelian fixed points in five dimensions, and one may be alarmed that the notion of the superconformal index is ill defined. However, the quantity Eq. (2.3), and its subsequent NS limit, is meaningful even for nonconformal theories, and it is this definition that we will use in the upcoming discussion. ${ }^{14}$

We have already seen that the existence of BPS instanton particles in 5D leads to index contributions with a new global fugacity, related to the topological charge. It is therefore natural to suspect that any 5D extension of the Schur-KS correspondence must involve a BPS quiver where at least one extra node, corresponding to the BPS instanton particle, is added.

Unlike four dimensions, the five-dimensional central charge is real, and the BPS states are divided into $C P T$ conjugate pairs. The states with the lowest possible charges (the "partonic" BPS states) comprise W-bosons and quarks, instanton solitons and magnetically charged BPS strings; see e.g. Ref. [12]. The existence of BPS strings makes the identification of the appropriate five-dimensional nonAbelian generalization of the BPS quiver subtle. ${ }^{15}$ However, for Abelian theories with $N_{f}$ flavors, BPS-string states are absent, and one can straightforwardly construct a 5D quiver comprising only of an instanton-particle node and a node for each of the $N_{f}$ flavors, with no arrows extending between them.

In the following section, we will show that the Abelian NS index can be reexpressed to match the trace of the KS operator for the corresponding 5D BPS quiver. We will also comment on the possible extension to non-Abelian gauge groups.

\section{A. Abelian theories}

For Abelian theories, the nonperturbative contribution is particularly simple. This allows for a straightforward reinterpretation of their NS index in terms of quiver data. The instanton partition function for the U(1) theory with $F$ flavors and Chern-Simons (CS) level $\kappa$ can be borrowed from Ref. [17] ${ }^{16}$ :

$$
Z_{\text {Nekrasov }}^{(k)}=\frac{(2 i)^{k(F-3)}}{k !} \times \int \prod_{I=1}^{k} \frac{\mathrm{d} \phi_{I}}{2 \pi} \frac{e^{i \kappa \phi_{I}}\left(\sin \frac{\phi_{I}}{2}\right)^{F} \prod_{I \neq J} \sin \frac{\phi_{I}-\phi_{J}}{2} \prod_{I, J} \sin \frac{\phi_{I}-\phi_{J}-2 i \gamma_{1}}{2}}{\prod_{i=1}^{N} \sin \frac{\phi_{I}-\alpha_{i}-i \gamma_{1}}{2} \sin \frac{-\phi_{I}+\alpha_{i}-i \gamma_{1}}{2} \prod_{I, J} \sin \frac{\phi_{I}-\phi_{J}-i \gamma_{1}-i \gamma_{2}}{2} \sin \frac{\phi_{I}-\phi_{J}-i \gamma_{1}+i \gamma_{2}}{2}} .
$$

\footnotetext{
${ }^{14}$ Having said that, "SU(1) theories" can exist at fixed points, since they correspond to $p q$ brane webs which can be collapsed to an intersection of five branes at a point. For instance, a pure "SU(1)" theory can be engineered in the NS-D5 intersection and corresponds to a pure $\mathrm{U}(1)$ gauge theory where the perturbative vector multiplet is removed. The leftover instanton sector, behaving as a hypermultiplet, then still remains. Thus, our Abelian computations can be understood in terms of these SU(1) theories, which often appear in quiver tails (e.g. Ref. [27]).

${ }^{15}$ For example, the results in Ref. [15] suggest that only BPS particles are important in reproducing the index.

${ }^{16}$ Compared to that reference, we have taken the limits of chemical potentials $m_{l} \rightarrow 1$ for simplicity.
} 
Recall that, as is well known, integrating out a massive flavor produces a shift to the CS level by a factor of $\Delta \kappa=\frac{\operatorname{sign}(m)}{2}$. As a consequence, odd $F$ requires a halfinteger $\kappa$. In order to take the NS limit of the index, we shall rewrite the above expression using the fugacities $p$ and $q$, as well as a gauge fugacity $u:^{17}$

$$
p=e^{-\left(\gamma_{1}+\gamma_{2}\right)}, \quad q=e^{-\left(\gamma_{1}-\gamma_{2}\right)}, \quad u=e^{i \alpha} .
$$

We will next consider specific cases by fixing the CS level and the number of flavors.

\section{Pure $\mathrm{U}(1)_{ \pm 1}$ theory}

Let us consider the pure $\mathrm{U}(1)$ theory. The bound in Ref. [11] requires $|\kappa|=0,1$. Setting $\kappa=1$, we find from Eq. (3.8)

$$
Z_{\text {Nekrasov }}^{(1)}=\frac{1}{u} \frac{p q}{(1-p)(1-q)}
$$

As discussed in Ref. [28], the instanton contributions should be invariant under a transformation that simultaneously sends $p \rightarrow 1 / q$ and $q \rightarrow 1 / p$; this is a transformation that is part of the superconformal group, under which the perturbative single-letter indices are invariant. However, as it stands, Eq. (3.10) is not invariant, and this presents a problem.

Recall that this issue typically arises whenever the corresponding brane configuration involves parallel external 5-brane legs. Indeed, in the case of $\mathrm{SU}(N)_{N}$ theories, the brane web includes a pair of external parallel NS5 branes. In the process of computing the instanton contributions by decoupling the $\mathrm{U}(1)$ factor from the $\mathrm{U}(N)_{N}$ theory, one finds that the naive result does not exhibit the expected $p \rightarrow 1 / q$ and $q \rightarrow 1 / p$ invariance. As first argued in Ref. [28], this noninvariance can be traced back to extra states left over from the naive truncation, which in the brane web description correspond to D-strings stretched between the parallel external NS5s. These can slide off to infinity and hence should not be taken into account.

The discarded contribution from Ref. [28] turns out to be precisely equal to the naive $\mathrm{U}(1)_{1}$ instanton piece (3.10). As a result, going over the same brane web argument, we conclude that (3.10) corresponds to states which should not be counted in the $5 \mathrm{D}$ theory. Upon removing them, we are left with $Z_{\text {Nekrasov }}^{(1)}=0$, so that the full instanton contribution in this case is simply unity. Note that, had we chosen the other sign for the CS level, $\kappa=-1$, we would have found the same function upon taking $u \rightarrow u^{-1}$. This is

\footnotetext{
${ }^{17}$ The chemical potentials $\gamma_{1}, \gamma_{2}$ appearing here are not related to the vectors $\gamma$ of the charge lattice $\Gamma$. We hope that this notation, which is compatible with the literature, will not cause confusion.
}

tantamount to exchanging instantons with anti-instantons, and the previous discussion goes through unchanged.

All in all, this theory has a trivial instanton sector; the index is purely perturbative and coincides with the Schur index of a four-dimensional $\mathcal{N}=2$ theory with the same gauge and flavor symmetries. Since there are no BPS particles in this rank-1 theory, the corresponding 5D BPS quiver is trivial. One can therefore simply express the answer in the general form of (3.1) by writing

$$
I_{\mathrm{KS}}=(q)_{\infty}^{2}
$$

\section{Pure $\mathrm{U}(1)_{0}$}

In four dimensions, the Schur index of the pure U(1) theory at zero CS level, $\kappa=0$, simply reads

$$
I^{4 \mathrm{D}}=\mathrm{PE}\left[-\frac{2 q}{(1-q)}\right]=\prod_{n=1}^{\infty}\left(1-q^{n}\right)^{2}=(q)_{\infty}^{2} .
$$

In turn, the BPS quiver in $4 \mathrm{D}$ is trivial, and therefore

$$
\operatorname{Tr}[\mathcal{O}]=1 .
$$

This fits the pattern of Ref. [5], since from (3.1) one also recovers that $I_{K S}=(q)_{\infty}^{2}$.

Let us now go to five dimensions. The exact index of the pure U(1) theory in 5D was worked out in Ref. [29]. This is

$I_{\mathrm{U}(1)_{0}}^{5 \mathrm{D}}=\mathrm{PE}\left[-\frac{p+q}{(1-p)(1-q)}+\frac{\sqrt{p q}\left(\mathfrak{q}+\mathfrak{q}^{-1}\right)}{(1-p)(1-q)}\right]$.

The first term is a free vector multiplet, while the second looks like a hypermultiplet with the gauge fugacities replaced by the instanton fugacities, $\mathfrak{q}$. We can therefore use (2.18) to infer the corresponding NS index

$$
I_{\mathrm{U}(1)_{0}}^{5 \mathrm{D}, \mathrm{NS}}=\mathrm{PE}\left[-\frac{2 q}{(1-q)}+\frac{\sqrt{q}\left(\mathfrak{q}+\mathfrak{q}^{-1}\right)}{(1-q)}\right] .
$$

As the instanton contribution is similar to that of a hypermultiplet, and in view of the fact that a free hypermultiplet contributes a flavor node to the BPS quiver [5], it is natural to suspect that there is a 5D BPS quiver description containing one node and yielding the correct 5D NS index.

In order to confirm this prediction, let us first pause to consider the nonperturbative part of the index (3.15). Concentrating on instantons alone, one can rewrite their contribution as

$$
\operatorname{PE}\left[\frac{\sqrt{q} \mathfrak{q}}{(1-q)}\right]=\sum_{m=0}^{\infty} \frac{(\sqrt{q} \mathfrak{q})^{m}}{\prod_{k=1}^{m}\left(1-q^{k}\right)}=E_{q}(-\mathfrak{q}),
$$

where in the last step we used Eq. (3.5). As an aside, it is interesting to observe that the above expression can be 
identified with the 5D ("K-theoretic") vortex partition function [30]. ${ }^{18}$ In fact, the NS limit of the full 5D index can be rewritten as

$I_{\mathrm{U}(1)_{0}}^{5 \mathrm{D}, \mathrm{NS}}=\prod_{n=1}^{\infty}\left(1-q^{n}\right)^{2} \prod_{n=0}^{\infty}\left(1-q^{n+\frac{1}{2}} \mathfrak{q}\right)^{-1} \prod_{n=0}^{\infty}\left(1-q^{n+\frac{1}{2}} \mathfrak{q}^{-1}\right)^{-1}$,

which with the help of (3.5) can in turn be massaged into

$$
\begin{aligned}
I_{\mathrm{U}(1)_{0}}^{5 \mathrm{D}, \mathrm{NS}} & =(q)_{\infty}^{2} E_{q}\left(-\mathfrak{q}^{-1}\right) E_{q}(-\mathfrak{q}) \\
& =(q)_{\infty}^{2} \operatorname{Tr}\left[E_{q}\left(X_{-\gamma_{f}}\right) E_{q}\left(X_{\gamma_{f}}\right)\right] .
\end{aligned}
$$

The above expression is consistent with it originating from a 5D rank-1 theory with a BPS quiver consisting of a single flavor node. The corresponding quantum torus algebra is commuting, and the formal variable $X_{\gamma_{f}}$ can be chosen such that $\operatorname{Tr}\left[X_{\gamma_{f}}\right]=-\mathfrak{q}$.

\section{3. $\mathrm{U}(1)_{-\frac{1}{2}}$ with one flavor}

Our next example is a U(1) theory with one flavor at CS level $\kappa=-\frac{1}{2}$. The 5D index reads

$$
I_{\mathrm{U}(1)-\frac{1}{2}}^{5 \mathrm{D}}=\int \frac{\mathrm{d} u}{u} Z_{\text {pert }} Z_{\text {nonpert }},
$$

where $u$ is the $\mathrm{U}(1)$ gauge fugacity and the perturbative contribution, after massaging (2.18), is given by

$Z_{\text {pert }}=\prod_{n=1}^{\infty}\left(1-q^{n}\right)^{2} \prod_{n=0}^{\infty}\left(1-q^{n+\frac{1}{2}} u\right)^{-1} \prod_{n=0}^{\infty}\left(1-q^{n+\frac{1}{2}} u^{-1}\right)^{-1}$.

In order to find the full nonperturbative contribution, given by the plethystic exponential of the one-instanton term, let us begin by looking at the latter. This is given by

$$
Z_{\mathrm{Nek}}^{(1)}=\frac{\sqrt{p q}}{(1-p)(1-q)}(1-u \sqrt{p q}) .
$$

As in the $|\kappa|=1$ case, the above expression is not invariant under a transformation which simultaneously sends $p \rightarrow$ $1 / q$ and $q \rightarrow 1 / p$. However, following Ref. [28] and introducing a correction factor,

$$
\Delta=\frac{q u}{(1-p)(1-q)},
$$

we can write a new one-instanton partition function in terms of

\footnotetext{
${ }^{18}$ The second part of Eq. (3.16) is to be compared with Eq. (3.16) of Ref. [30] or its generalization Eq. (2.40).
}

$$
Z_{\mathrm{Nek}}^{(1)}=Z_{\mathrm{Nek}}^{(1)}+\Delta=\frac{\sqrt{p q}}{(1-p)(1-q)} \text {. }
$$

This would suggest that the correct instanton sector contribution for $F=1$ is the same as for the $F=0$ case,

$$
Z_{\text {nonpert }}=\operatorname{PE}\left[\frac{\sqrt{p q}}{(1-p)(1-q)}\left(\mathfrak{q}+\mathfrak{q}^{-1}\right)\right] .
$$

By expanding to arbitrary order in the $q$ fugacity, it is straightforward to check that the NS index is equivalent to

$$
\begin{aligned}
I_{\mathrm{U}(1)_{-\frac{1}{2}}}^{\mathrm{D}, \mathrm{NS}}= & (q)_{\infty}^{2} \sum_{k_{1}, k_{2}, r_{1}, r_{2}=0}^{\infty} \\
& \times \frac{(-1)^{k_{1}+k_{2}+r_{1}+r_{2}} q^{\frac{k_{1}+k_{2}+r_{1}+r_{2}}{2}}(-\mathfrak{q})^{r_{2}-r_{1}} \delta_{k_{1}, k_{2}}}{(q)_{k_{1}}(q)_{k_{2}}(q)_{r_{1}}(q)_{r_{2}}} \\
= & (q)_{\infty}^{2} \operatorname{Tr}\left[E_{q}\left(X_{-\gamma_{f}}\right) E_{q}\left(X_{-\gamma}\right) E_{q}\left(X_{\gamma_{f}}\right) E_{q}\left(X_{\gamma}\right)\right] .
\end{aligned}
$$

In complete analogy with our previous discussion, the interpretation of this result in the language of Ref. [5] would be that the instanton provides a flavor charge $\gamma_{f}$, in addition to the charge lattice vector for the hypermultiplet, $\gamma$. This is consistent with having a 5D BPS quiver involving two nodes and no adjoining arrows.

\section{Maximally SUSY theory}

Consider adding to the U(1) vector multiplet a hypermultiplet in the adjoint representation. This is the content of the maximally supersymmetric (SUSY) theory. ${ }^{19}$ One might naively think that the adjoint hypermultiplet decouples and as a result that the instanton contribution is simply that of the pure $\mathrm{U}(1)$ theory. This is, however, not the case, as the noncommutative deformation regulating the Nekrasov partition function couples zero modes of the $\mathrm{U}(1)$ adjoint hypermultiplet to the instantons. In fact, it turns out [31] that the instanton contribution is

$$
\begin{aligned}
Z_{\text {inst }} & =\operatorname{PE}\left[\sum_{k=1}^{\infty} \mathfrak{q}^{k} z_{\mathrm{sp}}\right] \quad \text { with } \\
z_{\mathrm{sp}} & =-\frac{p+q}{(1-p)(1-q)}+2 \frac{\sqrt{p q}}{(1-p)(1-q)} .
\end{aligned}
$$

As stressed in Ref. [31], $z_{\mathrm{sp}}$ is equal to the contribution of a six-dimensional (6D) tensor multiplet. This constitutes a nontrivial check for the conjectured UV self-completion of the maximally SUSY 5D theory into the $(2,0)$ theory [32]. Note that the expression for $z_{\mathrm{sp}}$ above is exactly that of an

\footnotetext{
${ }^{19}$ Although this theory has $\mathcal{N}=2$ supersymmetry, we can still study it using $5 \mathrm{D} \mathcal{N}=1$ tools.
} 
Abelian vector plus an adjoint hypermultiplet. The latter is the full perturbative contribution of the 5D maximally SUSY theory, i.e.

$$
Z_{\text {pert }}=\operatorname{PE}\left[z_{\mathrm{sp}}\right] .
$$

Moreover, in the NS limit, one can reexpress

$\operatorname{PE}\left[\mathfrak{q}^{k} f_{H}\right]=\left(\prod_{m=0}^{\infty}\left(1-\mathfrak{q}^{k} q^{m+\frac{1}{2}}\right)^{-1}\right)^{2}=\left(E_{q}\left(-\mathfrak{q}^{k}\right)\right)^{2}$,

while

$$
\operatorname{PE}\left[\mathfrak{q}^{k} f_{V}\right]=\prod_{m=0}^{\infty}\left(1-\mathfrak{q}^{k} q q^{m}\right)^{2}=\left(\mathfrak{q}^{k} q ; q\right)^{2},
$$

where $(a ; b)$ stands for the q-Pochhammer symbol. ${ }^{20}$ The full index is given by

$$
I_{\mathrm{U}(1)}^{\mathrm{MaxSUSY}}=Z_{\text {pert }} Z_{\text {inst }} \bar{Z}_{\text {inst }},
$$

where the overline implies an inversion of the instanton fugacity. This prescription-which we stress is just the direct implementation of the results of Ref. [17] and strongly supported by nontrivial checks, including the emergence of the enhanced flavor symmetries in the case of $E_{N_{f}+1}$ theories-amounts to writing

$$
\bar{Z}_{\text {inst }}=\operatorname{PE}\left[\sum_{k=1}^{\infty} \mathfrak{q}^{-k} z_{\mathrm{sp}}\right]
$$

and Eq. (3.30) can be nicely repackaged into ${ }^{21}$

$$
I_{\mathrm{U}(1)}^{\mathrm{MaxSUSY}}=\prod_{k=-\infty}^{\infty}\left(\mathfrak{q}^{k} q ; q\right)^{2} E_{q}\left(-\mathfrak{q}^{-k}\right) E_{q}\left(-\mathfrak{q}^{k}\right) .
$$

This expression does not have a strict 5D BPS quiver interpretation. However, its form is rather suggestive: the collection of instantons corresponds to BPS states at threshold associated with the Kaluza-Klein modes that uplift the theory to 6D [31]. As such, one may expect that these would provide an infinite tower of flavor nodes, each parametrized by integer multiples of a fundamental charge, $\mathfrak{q}^{n}$, which is what we seem to find. However, the q-Pochhammer symbol, expected to arise from the

\footnotetext{
${ }^{20}$ The $(a ; b)$ q-Pochhammer symbol is defined as $(a ; b):=\prod_{j=0}^{\infty}\left(1-a b^{j}\right)$.

${ }^{21}$ Note that, by taking into account Eqs. (3.26), (3.27), and (3.31) and naively resumming the instanton expansions, it looks like the total partition function is $\mathrm{PE}[0]=1$. However, this conclusion is incorrect, since for this to happen each series is implicitly resummed in a different regime, while here $|\mathfrak{q}|=1$.
}

vector-multiplet contribution, also depends on $\mathfrak{q}^{n}$. It is tempting to speculate that this is due to the flavor fugacity combinations $\mathfrak{q}^{n}$ being remnants of a $6 \mathrm{D}$ Lorentz fugacity.

\section{B. Toward non-Abelian theories}

It is natural to ask whether there exists a non-Abelian extension of the correspondence between the NS index of a 5D SCFT and the trace of the KS operator for an associated BPS quiver, but we have thus far not been successful in constructing any such examples. Having a closed-form expression for the nonperturbative part of the non-Abelian NS index - the perturbative part reduces trivially to the 4D Schur index-would be helpful in pursuing this direction. Although the NS limit of the Abelian K-theoretic Nekrasov partition function coincides with the K-theoretic vortex partition function - cf. under Eq. (3.16) — explicitly applying our prescription (2.12) to non-Abelian gauge groups quickly produces an answer which disagrees with the $\mathfrak{q}$-expansion of any K-theoretic vortex partition function.

However, there may be another way forward using dualities. The instanton partition function-the 4D limit of the non-Abelian 5D Nekrasov partition function ${ }^{22}$-has a well-defined NS limit, originally discussed in Refs. [13,25], of which our prescription (2.12) is a natural generalization. As can be seen by expanding in the instanton fugacity, and simultaneously for small but nonzero $\epsilon_{1}, \epsilon_{2}$, Eq. (2.20) becomes

$$
\begin{aligned}
Z_{\mathrm{Nek}}^{\mathrm{NS}} \rightarrow & 1+\mathfrak{q}_{\epsilon_{2} \rightarrow 0} \epsilon_{2} Z_{\text {inst }}^{(1)} \\
& +\lim _{\epsilon_{2} \rightarrow 0} \mathfrak{q}^{2}\left(\frac{\epsilon_{2}\left(\epsilon_{2}-1\right)}{2}\left(Z_{\text {inst }}^{(1)}\right)^{2}+\epsilon_{2} Z_{\text {inst }}^{(2)}\right)+O\left(\mathfrak{q}^{3}\right) \\
= & \exp \left[\lim _{\epsilon_{2} \rightarrow 0} \epsilon_{2} \log Z_{\text {inst }}\left(\mathfrak{q} ; \epsilon_{1}, \epsilon_{2}\right)\right],
\end{aligned}
$$

precisely the expression appearing in Ref. [13]. In that reference, the resultant partition function was identified as the nonperturbative contribution to the twisted superpotential for some associated two-dimensional theory. Subsequently, the authors of Ref. [33] also linked the full 2D twisted superpotential-the NS limits of the full perturbative plus nonperturbative partition functions of the 4D theory-with the twisted superpotential for a different, dual 2D theory. Interestingly, the latter theory can in certain cases-e.g. the Abelian example-be interpreted as the world volume description for a $2 \mathrm{D}$ defect in the Higgs branch of the original 4D theory. The partition

\footnotetext{
${ }^{22}$ This is known as the "homological limit" (see e.g. Ref. [30] and references therein), and in notation where one has made explicit the dependence of the fugacities on the Euclideanized time radius, $p=e^{-\beta \epsilon_{1}}, q=e^{-\beta \epsilon_{2}}$, corresponds to taking $\beta \rightarrow 0$. In this limit, the full "K-theoretic" version of the Nekrasov partition function we have been using thus far reduces to the 4D instanton partition function.
} 
function for these defects is the well-known vortex partition function, which has a natural K-theoretic lift up to $5 \mathrm{D} .^{23} \mathrm{It}$ would be interesting to closely study similar 5D $\rightarrow$ 4D $\rightarrow$ $2 \mathrm{D} \rightarrow 2 \mathrm{D} \rightarrow 4 \mathrm{D} \rightarrow 5 \mathrm{D}$ chains for more complicated theories. This in turn could lead to identifying closed-form expressions for the NS limits of non-Abelian instanton contributions and shed light on how to proceed with the non-Abelian extension of the NS-KS correspondence presented in this section.

Another closely related task would be to investigate whether the 5D NS index we have defined admits an alternative (and possibly simpler) description associated with some lower-dimensional structure, along the lines of

\footnotetext{
${ }^{23}$ The details of this K-theoretic vortex partition function depend on how the embedding of the defect breaks the gauge group, i.e. on the choice of Levi subgroup; see e.g. Ref. [30] and references therein for details. See also Ref. [34] for new results on the calculation of $5 \mathrm{D}$ instanton partition functions in the presence of defects.
}

Ref. [4]. In this respect, the similarity of our prescription to the large orbifold limit of Ref. [14] may hint toward such a connection. We will leave the answers to these questions as open problems for future research.

\section{ACKNOWLEDGMENTS}

We would like to thank M. Buican and J. Hayling for helpful discussions and comments. A.P. and D. R. G. would like to acknowledge the CRST at Queen Mary University of London for hospitality during various stages of this work and the associated support of the COST Action MP1210 STSM. C. P. is supported by the Royal Society through a University Research Fellowship. A.P. and D. R. G. are partly supported by the Spanish Government Grant No. MINECO-13-FPA2012-35043C02-02. In addition, they acknowledge financial support from the Ramon y Cajal Grant No. RYC-2011-07593 as well as the EU CIG Grant No. UE-14-GT5LD2013618459. The work of A.P. is funded by the Asturian Government SEVERO OCHOA Grant No. BP14-003.
[1] C. Romelsberger, Counting chiral primaries in $N=1$, $d=4$ superconformal field theories, Nucl. Phys. B747, 329 (2006); J. Kinney, J. M. Maldacena, S. Minwalla, and S. Raju, An index for 4 dimensional super conformal theories, Commun. Math. Phys. 275, 209 (2007).

[2] J. Bhattacharya, S. Bhattacharyya, S. Minwalla, and S. Raju, Indices for Superconformal Field theories in 3, 5 and 6 dimensions, J. High Energy Phys. 02 (2008) 064.

[3] A. Gadde, L. Rastelli, S. S. Razamat, and W. Yan, Gauge theories and Macdonald polynomials, Commun. Math. Phys. 319, 147 (2013).

[4] C. Beem, M. Lemos, P. Liendo, W. Peelaers, L. Rastelli, and B. C. van Rees, Infinite Chiral Symmetry in Four Dimensions, Commun. Math. Phys. 336, 1359 (2015); C. Beem, L. Rastelli, and B.C. van Rees, $\mathcal{W}$ symmetry in six dimensions, J. High Energy Phys. 05 (2015) 017.

[5] C. Córdova and S.-H. Shao, Schur Indices, BPS Particles, and Argyres-Douglas Theories, J. High Energy Phys. 01 (2016) 040.

[6] J. Bourdier, N. Drukker, and J. Felix, The exact Schur index of $\mathcal{N}=4$ SYM, J. High Energy Phys. 11 (2015) 210; The $\mathcal{N}=2$ Schur index from free fermions, J. High Energy Phys. 01 (2016) 167; N. Drukker, The $\mathcal{N}=4$ Schur index with Polyakov loops, J. High Energy Phys. 12 (2015) 012.

[7] M. Buican and T. Nishinaka, On the superconformal index of Argyres-Douglas theories, J. Phys. A 49, 015401 (2016).

[8] S. Cecotti, J. Song, C. Vafa, and W. Yan, Superconformal Index, BPS Monodromy and Chiral Algebras, arXiv: 1511.01516 .
[9] N. Seiberg, Five-dimensional SUSY field theories, nontrivial fixed points and string dynamics, Phys. Lett. B 388, 753 (1996).

[10] D. R. Morrison and N. Seiberg, Extremal transitions and five-dimensional supersymmetric field theories, Nucl. Phys. B483, 229 (1997); M. R. Douglas, S. H. Katz, and C. Vafa, Small instantons, Del Pezzo surfaces and type I-prime theory, Nucl. Phys. B497, 155 (1997).

[11] K. A. Intriligator, D. R. Morrison, and N. Seiberg, Five-dimensional supersymmetric gauge theories and degenerations of Calabi-Yau spaces, Nucl. Phys. B497, 56 (1997).

[12] O. Aharony, A. Hanany, and B. Kol, Webs of (p,q) fivebranes, five-dimensional field theories and grid diagrams, J. High Energy Phys. 01 (1998) 002.

[13] N. A. Nekrasov and S. L. Shatashvili, Proceedings, 16th International Congress on Mathematical Physics (ICMP09): Prague, Czech Republic, August 3-8, 2009 (World Scientific, Singapore, 2010), p. 708.

[14] N. Mekareeya and D. Rodriguez-Gomez, 5d gauge theories on orbifolds and 4d 't Hooft line indices, J. High Energy Phys. 11 (2013) 157.

[15] A. Iqbal and C. Vafa, BPS Degeneracies and Superconformal Index in Diverse Dimensions, Phys. Rev. D 90, 105031 (2014).

[16] V. Pestun, Localization of gauge theory on a four-sphere and supersymmetric Wilson loops, Commun. Math. Phys. 313, 71 (2012).

[17] H.-C. Kim, S.-S. Kim, and K. Lee, 5-dim Superconformal Index with Enhanced En Global Symmetry, J. High Energy Phys. 10 (2012) 142. 
[18] D. Gaiotto and H.-C. Kim, Duality walls and defects in 5d $N=1$ theories, arXiv:1506.03871.

[19] N. Lambert, C. Papageorgakis, and M. SchmidtSommerfeld, Instanton operators in five-dimensional gauge theories, J. High Energy Phys. 03 (2015) 019.

[20] Y. Tachikawa, Instanton operators and symmetry enhancement in $5 \mathrm{~d}$ supersymmetric gauge theories, Prog. Theor. Exp. Phys. 2015, 43 B06 (2015).

[21] S. Terashima, Supersymmetric gauge theories on $S^{4} \times S^{1}$, Phys. Rev. D 89, 125001 (2014).

[22] A. Pini, D. Rodriguez-Gomez, and J. Schmude, Rigid supersymmetry from conformal supergravity in five dimensions, J. High Energy Phys. 09 (2015) 118.

[23] O. Bergman and D. Rodriguez-Gomez, A note on instanton operators, instanton particles, and supersymmetry, J. High Energy Phys. 05 (2016) 068.

[24] N. Nekrasov and A. Okounkov, Seiberg-Witten theory and random partitions, Progress of mathematics 244, 525 (2006); N. A. Nekrasov, Seiberg-Witten prepotential from instanton counting, Adv. Theor. Math. Phys. 7, 831 (2003).

[25] N. Nekrasov and V. Pestun, Seiberg-Witten geometry of four dimensional $\mathrm{N}=2$ quiver gauge theories, arXiv: 1211.2240; N. Nekrasov, V. Pestun, and S. Shatashvili, Quantum geometry and quiver gauge theories, arXiv: 1312.6689.

[26] S. Cecotti, A. Neitzke, and C. Vafa, R-Twisting and 4d/2d Correspondences, arXiv:1006.3435.
[27] O. Bergman and G. Zafrir, Lifting 4d dualities to 5d, J. High Energy Phys. 04 (2015) 141; K. Ohmori, H. Shimizu, Y. Tachikawa, and K. Yonekura, $6 \mathrm{~d} \mathcal{N}=(1,0)$ theories on $T^{2}$ and class S theories: Part I, J. High Energy Phys. 07 (2015) 014.

[28] O. Bergman, D. Rodríguez-Gómez, and G. Zafrir, Discrete $\theta$ and the 5d superconformal index, J. High Energy Phys. 01 (2014) 079.

[29] D. Rodríguez-Gómez and G. Zafrir, On the 5d instanton index as a Hilbert series, Nucl. Phys. B878, 1 (2014).

[30] T. Dimofte, S. Gukov, and L. Hollands, Vortex counting and Lagrangian 3-manifolds, Lett. Math. Phys. 98, 225 (2011).

[31] H.-C. Kim, S. Kim, E. Koh, K. Lee, and S. Lee, On instantons as Kaluza-Klein modes of M5-branes, J. High Energy Phys. 12 (2011) 031.

[32] M. R. Douglas, On $D=5$ super Yang-Mills theory and (2, 0) theory, J. High Energy Phys. 02 (2011) 011; N. Lambert, C. Papageorgakis, and M. Schmidt-Sommerfeld, M5-branes, D4-branes and quantum 5D super-Yang-Mills, J. High Energy Phys. 01 (2011) 083.

[33] N. Dorey, S. Lee, and T. J. Hollowood, Quantization of integrable systems and a 2d/4d duality, J. High Energy Phys. 10 (2011) 077; H.-Y. Chen, N. Dorey, T. J. Hollowood, and S. Lee, A new 2d/4d duality via integrability, J. High Energy Phys. 09 (2011) 040.

[34] H.-C. Kim, Line defects and 5d instanton partition functions, J. High Energy Phys. 03 (2016) 199. 